The Unified Protocol as an Internet-based Intervention for Emotional Disorders:

\title{
Randomized Controlled Trial
}

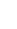

(1)

Carmen Schaeuffele ${ }^{1}$, Sophie Homeyer ${ }^{1}$, Luis Perea $^{1}$, Lisa Scharf $^{1}$, Ava Schulz $^{2}$, Christine

(1)

$$
\text { Knaevelsrud }^{1} \text {, Babette Renneberg }{ }^{1} \text {, and Johanna Boettcher }{ }^{1,3}
$$

\footnotetext{
${ }^{1}$ Department of Education and Psychology, Freie Universität Berlin, Berlin, Germany ${ }^{2}$ Department of Psychiatry, Psychotherapy and Psychosomatics, University of Zurich, Switzerland

${ }^{3}$ Clinical Psychology and Psychotherapy, Psychologische Hochschule Berlin, Berlin, Germany
}

Please note: This is a preprint version of the manuscript and has not been peer-reviewed

Address for Correspondence: Carmen Schaeuffele, Freie Universität Berlin, Department of Education and Psychology, Clinical Psychology and Psychotherapy, Habelschwerdter Allee 45, 14195 Berlin, Germany, email: carmen.schaeuffele@ fu-berlin.de

Funding: This research did not receive any specific grant from funding agencies in the public, commercial, or not-for-profit sectors.

Conflict of interest: The authors do not have any conflict of interest to report. 


\section{Abstract}

The Unified Protocol (UP) as a transdiagnostic intervention has primarily been applied in the treatment of anxiety disorders and in face-to-face-settings. The current study investigated the efficacy of a 10-week Internet-based adaptation of the UP for anxiety, depressive, and somatic symptom disorders. The trial was registered under DRKS00014820 at the German Clinical Trial Registry, DRKS. $N=129$ participants were randomized to treatment or waitlist control. Significant treatment effects were found for symptom distress, satisfaction with life, positive/negative affect and markers of anxiety, depression, and somatic symptom burden (within-group Hedges' $g=0.32-1.38$ and between-group $g=0.20-1.11$ ). Treatment gains were maintained at 1- and 6-month-follow-up. Subgroup analyses showed comparable effects in participants with anxiety and depressive disorders. $26.6 \%$ dropped out of treatment and $35.38 \%$ did not provide post-treatment assessments.

The results strengthen the application of the UP as an Internet-based treatment for alleviating symptom distress across emotional disorders. More research on the applicability for single disorders is needed and avenues to improve adherence and attrition rates should be explored.

Keywords: transdiagnostic; Internet-based cognitive behavior therapy; Unified Protocol, emotional disorders; guided self-help 


\section{The Unified Protocol as an Internet-Based Intervention for}

\section{Emotional Disorders: Randomized Controlled Trial}

About a third of the German population fulfilled criteria for at least one mental disorder in the previous 12 months [1]. The emotional disorders, subsuming anxiety, depressive, and related disorders, are most common and highly comorbid [1]. While comorbidity results in greater severity, impairment, and chronicity [2-4], the majority of evidence-based treatments is focused on treating single disorders [5]. Transdiagnostic treatments, that simultaneously target several disorders, address comorbidity and have the potential to facilitate the dissemination of evidence-based treatments [6]. Transdiagnostic approaches have been applied successfully in face-to-face $[7,8]$ and Internet-based settings $[9,10]$.

While there is a growing evidence base for transdiagnostic treatments, transdiagnostic treatments encompass heterogeneous approaches that vary considerably in their foundation in theory [11]. In addition, the majority of treatments are applied exclusively in Internet-based or face-to-face settings [7-10]. However, this impedes dissemination and efforts to integrate online interventions with face-to-face therapy. Instead, it seems reasonable to concentrate on effective transdiagnostic treatments with clear assumptions about "shared mechanisms" between disorders to target a wider range of disorders and adaptability across different settings and populations, like the Unified Protocol (UP) $[6,12,13]$. The Unified Protocol is a transdiagnostic treatment for emotional disorders that targets dysfunctional reactions towards emotions, originally developed for the face-to-face setting [14]. The UP led to moderate to large effect sizes compared to waitlist and showed equivalence to gold-standard singledisorder protocols for mixed anxiety disorders $[15,16]$. The UP has been delivered over the Internet as a 10-week guided intervention in a first preliminary study for anxiety and 
depression [17]. While encouraging, the study sample seemed less impaired than in face-toface RCTs and the trial suffered from high attrition.

The high comorbidity, overlap, and shared mechanisms between emotional disorders, raises the question of the UP's applicability beyond anxiety disorders. So far, the majority of research efforts have been concentrated on anxiety and the evidence base beyond anxiety disorders is still limited [12]. While a meta-analysis found that the UP led to medium to large effects across anxiety and depression [18], the trials that did investigate the UP for primary depression are single case studies and open trials [14,19-23]. The Internet-based RCT included primary depression but did not report findings for this diagnostic group separately [17]. The third most prevalent group of emotional disorder are somatic symptom disorders [1]. Despite their prevalence in primary care, their high medical health care utilization as well as high risk of chronicity and disability [24], somatic symptom disorders have not been investigated within the UP framework.

Our goal for this study was to extend the evidence-base for the UP in an Internet-based setting for disorders beyond anxiety disorders. We were interested in both overall and differential efficacy of the UP in the context of a guided self-help intervention for emotional disorders, namely anxiety, depressive, and somatic symptom disorders.

\section{Method}

\section{Design}

We compared the Internet-based intervention based on the UP to waitlist control in an RCT to explore its efficacy. The trial was registered under DRKS00014820 at DRKS.

Participation was free of charge and not reimbursed.

\section{Participants}

We included participants if they (a) were over 18 years, (b) had a sufficient knowledge of German, (c) had Internet access, (d) had a stable dose of medication over the preceding 
three months, and (e) had one of the following primary diagnoses: panic disorder, agoraphobia, social anxiety disorder, generalized anxiety disorder, persistent depressive disorder, major depressive disorder, illness anxiety disorder, and somatic symptom disorder. We excluded participants from the study if they (a) currently experienced symptoms of a psychotic, bipolar, or substance use disorder, (b) were suicidal, or (c) were currently in psychotherapy.

We recruited participants in mental health online forums as well as via online advertising. Participants were directed to a study website with detailed information as well as a registration and consent form. After obtaining written informed consent, we activated an online screening with demographic and self-report measures. If participants' ratings reached one or more pre-defined cut-offs (PHQ-9>10, LSAS >30, GAD-7>10, PAS>10, SHAI>18), we invited them to a structured clinical interview via telephone to determine inclusion and exclusion diagnoses [25]. A detailed description of the recruitment procedure is shown in the flow diagram in Fig 1. Recruitment commenced in December 2018 and ended in February 2020.

Fig 1. Consort Flow Chart.

----- please insert Fig.1 here -----

\section{Intervention}

The 10-week guided transdiagnostic intervention is an adaptation of the UP for Internet-based use. We shortened and simplified the protocol, while retaining the core concepts of the UP (see Table 1). The sequence and contents of the modules were fixed for all participants. We recommended participants to complete one module per week, but participants were free to work at their own pace. Weekly asynchronous guidance was provided by the first and last author as well as by 16 graduate students of clinical psychology. The master students were 
trained in a 1-day workshop and biweekly supervision meetings were held throughout the trial to ensure adherence. On average, online therapists spent $24.44(S D=7.92)$ minutes per week on every participant.

\section{Table 1. Overview of Guided Intervention.}

\begin{tabular}{|c|c|c|}
\hline Week & Name & Description \\
\hline 1 & $\begin{array}{l}\text { Motivation and } \\
\text { goal setting }\end{array}$ & $\begin{array}{l}\text { Participants learn about motivation and goal setting. They fill in a } \\
\text { decisional balance sheet and set 1-3 goals for treatment. }\end{array}$ \\
\hline 2 & $\begin{array}{l}\text { Understanding } \\
\text { Emotions }\end{array}$ & $\begin{array}{l}\text { Participants learn about emotions and the difference between } \\
\text { cognition, emotion, and behavior. They record their emotional } \\
\text { experiences and reflect the short- and long-term consequences of their } \\
\text { behavior. }\end{array}$ \\
\hline 3 & Mindfulness & $\begin{array}{l}\text { Participants learn about primary and secondary emotions and the } \\
\text { benefits of mindful emotion awareness. They practice a selection of } \\
\text { mindfulness exercises. }\end{array}$ \\
\hline 4 & $\begin{array}{l}\text { Cognitive } \\
\text { Flexibility I }\end{array}$ & $\begin{array}{l}\text { Participants learn about the relationship of thoughts and emotions, } \\
\text { automatic thoughts and thinking traps. They challenge their thoughts } \\
\text { by finding alternative thoughts. }\end{array}$ \\
\hline 5 & $\begin{array}{l}\text { Cognitive } \\
\text { Flexibility II }\end{array}$ & $\begin{array}{l}\text { Participants learn about thoughts about emotions and core beliefs. They } \\
\text { continue with challenging their thoughts. }\end{array}$ \\
\hline 6 & $\begin{array}{l}\text { Countering } \\
\text { Avoidance }\end{array}$ & $\begin{array}{l}\text { Participants learn about avoidance and countering avoidance. They } \\
\text { reflect on their avoidance tendencies and record their experience with } \\
\text { countering avoidance. }\end{array}$ \\
\hline 7 & $\begin{array}{l}\text { Interoceptive } \\
\text { Exposure }\end{array}$ & $\begin{array}{l}\text { Participants learn about the effect of physical sensations. They induce } \\
\text { physical sensations with video-guided interoceptive exposures and own } \\
\text { exercises. }\end{array}$ \\
\hline 8 & $\begin{array}{l}\text { Emotion } \\
\text { Exposure }\end{array}$ & $\begin{array}{l}\text { Participants set a hierarchy of "difficult situations" and conduct } \\
\text { emotion exposures in vivo and sensu. }\end{array}$ \\
\hline 9 & $\begin{array}{l}\text { Emotion } \\
\text { Exposure II }\end{array}$ & $\begin{array}{l}\text { Participants continue to expose themselves to emotion-inducing } \\
\text { situations and images. }\end{array}$ \\
\hline 10 & $\begin{array}{l}\text { Relapse } \\
\text { Prevention }\end{array}$ & $\begin{array}{l}\text { Participants reflect on achievements and compile a training schedule } \\
\text { for when treatment is over. }\end{array}$ \\
\hline
\end{tabular}

\section{Outcome Measures}

We interviewed participants with a structured clinical interview via telephone prior to randomization. After 10 weeks, participants of the treatment group were interviewed again to 
online platform. We assessed the primary outcome measure (BSI-18) and transdiagnostic secondary outcome measures at baseline, mid- and post-treatment as well at 1-month and 6month follow-up. We assessed all disorder-specific measures at baseline and post-treatment. Negative effects and treatment satisfaction were collected at post-treatment.

\section{Structured Clinical Interview}

We interviewed participants with the Diagnostic Interview for Mental Disorders (DIPS) $[25,26]$, a structured clinical interview for DSM-5, via telephone to determine diagnostic status of participants. The DIPS has good psychometric properties [25]. Interviewers were the first author as well as six master students of clinical psychology who were trained and supervised. Ten interviews were audiotaped and rated by an independent second rater. Interrater reliability ranged between $95.12 \%$ (anxiety disorders) and $100 \%$ accordance (all other diagnostic groups).

\section{Primary Outcome}

Primary outcome was symptom distress, as measured by the Brief Symptom Inventory 18 (BSI-18). The BSI-18 [27,28] is an 18-item short-version of the Symptom-Checklist-90-R and includes three subscales (anxiety, depression, and somatization). The BSI-18 shows high internal reliability and overall satisfactory psychometric properties (Derogatis \& Fitzpatrick, 2004; Franke et al., 2011; Prinz et al., 2013).

\section{Transdiagnostic Secondary Outcome Measures}

We assessed positive and negative affect with the Positive and Negative Affect Schedule (PANAS). The PANAS [31,32] measures positive and negative affect with two 10item scales. Both scales show high internal consistency and are quasi-independent (Watson et al., 1988). Satisfaction with life was assessed with the Satisfaction with Life Scale (SWLS). The SWLS $[33,34]$ is a 5-item measure of life satisfaction. Internal consistency and re-test reliability are high. 


\section{Disorder-specific Secondary Outcome Measures}

We assessed depressive symptoms with the Patient Health Questionnaire (PHQ-9). The PHQ-9 $[35,36]$ is a 9-item screening instrument for depression with satisfactory psychometric properties (Kroenke \& Spitzer, 2002; Lowe, 2004). Symptoms of generalized anxiety were assessed with the 7-item Generalized Anxiety Disorder Screener (GAD-7) [37,38]. The GAD-7 is unidimensional and internally reliable. The Liebowitz Social Anxiety Schedule (LSAS) was used to assess social anxiety. The LSAS $[39,40]$ is 24 -item measure of social anxiety assessing fear/anxiety and avoidance. Internal consistency of the two subscales as well as the total scale is high and the scale exhibits good psychometric properties [41]. We assessed panic and agoraphobia with the 13-item Panic and Agoraphobia Scale (PAS) [42,43]. The PAS assesses panic attacks and agoraphobic avoidance as well as related concerns. Internal reliability as well as psychometric properties are good. Health anxiety was assessed with the 18-item Short Health Anxiety Inventory (SHAI) $[44,45]$. The SHAI has a two-factor structure and assesses health anxiety with 14 items and perceived negative consequences of being ill with four items. The SHAI has good psychometric properties. We used the 15 -item Patient Health Questionnaire (PHQ-15) to assess somatic symptom burden (Gräfe et al., 2004; Kroenke et al., 2002). Thirteen items represent the most common somatic symptoms and participants rate the extent to which they are bothered by these symptoms. Two items assess sleep disturbance and tiredness and are also included in the PHQ-9 and scored accordingly. Their ratings were obtained in the PHQ-9 assessment and aggregated to a total score. Internal consistency and psychometric properties are good.

\section{Negative Effects and Satisfaction with Treatment}

We assessed negative effects of treatment with the Negative Effects Questionnaire (NEQ) [48], a 20-item measure of the occurrence and characteristics of negative effects following treatment. Each item is rated in three steps: if participants agree that the negative effect occurred, then they are asked to rate the negative impact of the negative effect and 
attribute it to treatment or other circumstances. We used the Client Satisfaction Questionnaire8 (CSQ-8) [49,50] to measure client satisfaction with treatment with 8 questions. The CSQ-8 is unidimensional and internally reliable [51].

\section{Sample Size}

Sample size was calculated using $\mathrm{g}^{*}$ power [52]. We hypothesized a large effect (Cohen's $d=0.8$ ) in favor of the intervention [15,21]. To detect this effect (one-sided t-test for independent samples, $\alpha=.05$ ) with a power of $80 \%$, a sample size of $n=42$ participants is required. With an assumed attrition rate of $15 \%$, we aimed at recruiting a sample size of $n=$ 60 per diagnostic group (anxiety, depressive, and somatic symptom disorder).

\section{Randomization Procedure}

We randomized participants to waitlist or treatment in a 1:1 ratio. We used stratified block randomization to ensure a balanced distribution of primary diagnosis groups (anxiety, depressive, and somatic symptom disorder as stratum) across treatment and waitlist. The allocation sequence was generated by an online random number generator and carried out by the online platform that also hosted the intervention. A total of $n=132$ participants were randomized: $n=60$ with a primary anxiety, $n=60$ with a primary depressive disorder, and $n$ = 12 with a primary somatic symptom disorder. Despite increased efforts, we failed to meet our original recruitment goal regarding somatic symptom disorder within the study's timeframe.

\section{Statistical Analysis}

We conducted all analyses using RStudio [53]. All analyses followed the intent-totreat framework. Group differences in demographics and baseline measures were tested using t-tests or Pearson's Chi-squared tests. Statistical significance was set at the 5\% level, and confidence intervals were calculated at the $95 \%$ confidence level. For the efficacy and followup analyses, we chose mixed effects models for their advantages in handling missing as well 
as longitudinal data. We fitted linear mixed effects models with the lmer-function from the lme4 package [54], using the lmerTest package to obtain p-values [55]. For the efficacy analysis, we included treatment, time, and the interaction of treatment and time as fixed and participants as random effects in the model. We also conducted the same analysis in a subgroup of primarily anxious and primarily depressed participants. To analyze whether treatments gains were maintained in the treatment group at 1- and 6-month follow up, we included time as fixed and participants as random effects in the model. We calculated effect sizes (Hedges' $g$ ) based on the estimated means and the pooled standard deviations of the observed means. Response was determined based on the reliable change index $(R C I=1.96)$ [56]. RCIs were calculated using the BSI-18's internal reliability $(\alpha=.91)[28,57]$. Since norm data from a clinical and general population was available $[28,58]$, we also calculated the Cutoff $C$ to determine recovery rates [56]. Individuals are recovered if they fall below the cutoff and show reliable improvement according to the RCI [56].

\section{Ethics}

The trial was approved by the ethics committee of the Department of Education and Psychology at Freie Universitaet Berlin, Germany (186/2018).

\section{Results}

\section{Demographics}

Demographic characteristics are displayed in Table 2. Groups did not differ in demographic variables.

Table 2. Demographic Characteristics of Participants at Baseline.

\begin{tabular}{|c|c|c|c|c|c|}
\hline \multirow[b]{2}{*}{ Demographic Variable } & \multirow[b]{2}{*}{$\begin{array}{l}\text { Treatment } \\
(n=65)\end{array}$} & \multirow[b]{2}{*}{$\begin{array}{l}\text { Waitlist } \\
(n=64)\end{array}$} & \multirow[b]{2}{*}{ Total } & \multicolumn{2}{|c|}{$\begin{array}{l}\text { Statistical test of } \\
\text { group difference }\end{array}$} \\
\hline & & & & $\mathrm{df} \quad \chi^{2 / t}$ & $p$ \\
\hline Gender & & & & .48 & .49 \\
\hline Male & $23(35.4 \%)$ & $18(28.1 \%)$ & $41(31.8 \%)$ & & \\
\hline Female & $42(64.6 \%)$ & $46(71.9 \%)$ & $88(68.2 \%)$ & & \\
\hline
\end{tabular}




\begin{tabular}{|c|c|c|c|c|c|c|}
\hline Age & & & & 127 & .18 & .86 \\
\hline Mean (SD) & $37.51(11.99)$ & $37.11(13.04)$ & $37.31(12.47)$ & & & \\
\hline Range & $18-67$ & $18-66$ & $18-67$ & & & \\
\hline Relationship & & & & 1 & 1.99 & .16 \\
\hline in a relationship & $40(61.5 \%)$ & $30(46.9 \%)$ & $70(54.3 \%)$ & & & \\
\hline single & $25(38.5 \%)$ & $34(53.1 \%)$ & $59(45.7 \%)$ & & & \\
\hline Highest Education & & & & 4 & 4.7 & .32 \\
\hline $\begin{array}{l}\text { up to } 9 \text { years of school } \\
\text { education }\end{array}$ & $4(6.2 \%)$ & $5(7.8 \%)$ & $9(7 \%)$ & & & \\
\hline Secondary school & $10(15.4 \%)$ & $11(17.2 \%)$ & $21(16.3 \%)$ & & & \\
\hline $\begin{array}{l}\text { College entrance } \\
\text { qualification }\end{array}$ & $16(24.6 \%)$ & $25(39.1 \%)$ & $41(31.8 \%)$ & & & \\
\hline College / university degree & $34(52.3 \%)$ & $22(34.4 \%)$ & $56(43.3 \%)$ & & & \\
\hline other & $1(1.5 \%)$ & $1(1.6 \%)$ & $2(1.6 \%)$ & & & \\
\hline Employment status & & & & 5 & 2.99 & .7 \\
\hline employed & $33(50.8 \%)$ & $29(45.3 \%)$ & $62(48.1 \%)$ & & & \\
\hline self-employed & $4(6.2 \%)$ & $5(7.8 \%)$ & $9(7 \%)$ & & & \\
\hline in education & $12(18.5 \%)$ & $17(26.6 \%)$ & $29(22.5 \%)$ & & & \\
\hline pensioned & $3(4.6 \%)$ & $5(7.8 \%)$ & $8(6.2 \%)$ & & & \\
\hline unemployed & $9(13.8 \%)$ & $6(9.4 \%)$ & $15(11.6 \%)$ & & & \\
\hline other & $4(6.2 \%)$ & $2(3.1 \%)$ & $6(4.7 \%)$ & & & \\
\hline Currently on medication & & & & 1 & 0 & .99 \\
\hline yes & $16(24.6 \%)$ & $16(25 \%)$ & $32(24.8 \%)$ & & & \\
\hline no & $49(75.4 \%)$ & $48(75 \%)$ & $97(75.2 \%)$ & & & \\
\hline Prior Psychotherapy & & & & 1 & 1.95 & .16 \\
\hline yes & $44(67.7 \%)$ & $51(79.7 \%)$ & $95(73.6 \%)$ & & & \\
\hline no & $21(32.3 \%)$ & $13(20.3 \%)$ & $34(26.4 \%)$ & & & \\
\hline Primary Diagnosis & & & & 9 & 5.66 & .77 \\
\hline Agoraphobia & $3(4.6 \%)$ & $4(6.3 \%)$ & $7(5.4 \%)$ & & & \\
\hline Generalized Anxiety & $9(13.8 \%)$ & $5(7.8 \%)$ & $14(10.8 \%)$ & & & \\
\hline Disorder & & & & & & \\
\hline Panic Disorder & $5(7.7 \%)$ & $5(7.8 \%)$ & $10(7.8 \%)$ & & & \\
\hline Social Anxiety Disorder & $13(20 \%)$ & $16(25 \%)$ & $29(22.5 \%)$ & & & \\
\hline Major Depressive Disorder & $14(21.5 \%)$ & $17(26.6 \%)$ & $31(24 \%)$ & & & \\
\hline Persistent Depressive & $15(23.1 \%)$ & $12(18.8 \%)$ & $27(20.9 \%)$ & & & \\
\hline Disorder & & & & & & \\
\hline Somatic Symptom Disorder & $2(3.1 \%)$ & $3(6.3 \%)$ & $5(3.9 \%)$ & & & \\
\hline Illness Anxiety Disorder & $4(6.2 \%)$ & $2(3.1 \%)$ & $6(4.7 \%)$ & & & \\
\hline Comorbidity & & & & & & \\
\hline 1 comorbid diagnosis & $51(78.5 \%)$ & $48(75 \%)$ & $99(76.7 \%)$ & & & \\
\hline 2 comorbid diagnoses & $27(41.5 \%)$ & $28(43.7 \%)$ & $55(42.6 \%)$ & & & \\
\hline 3 comorbid diagnoses & $18(27.7 \%)$ & $18(28.1 \%)$ & $36(27.9 \%)$ & & & \\
\hline 4 comorbid diagnoses & $12(18.5 \%)$ & $10(15.6 \%)$ & $22(17.1 \%)$ & & & \\
\hline 5 comorbid diagnoses & $5(7.7 \%)$ & $4(6.2 \%)$ & $9(6.9 \%)$ & & & \\
\hline
\end{tabular}

\section{Treatment Adherence, Usage \& Attrition}

233 six or more sessions within ten weeks, which is equal to being exposed to all core elements of

234 therapy (mindfulness, cognitive flexibility, and emotion avoidance). Non-completers 
completed five or less sessions within ten weeks and/or communicated treatment termination. Accordingly, $n=47$ (73.4\%) were completers and $n=17$ (26.6\%) were non-completers. $n=27$ $(42.2 \%)$ completed all 10 modules. Participants on average logged in $85.3(S D=39.2)$ times and spent $57.4(S D=34.9)$ hours on the platform. However, this does not reflect actual usage time, as the website did not automatically log participants out. Participants completed on average $7.22(S D=3.15)$ modules, $36.17(S D=29.04)$ exercises, $0.41(S D=0.3)$ exercises per login and wrote $8.05(S D=7.97)$ messages. In the treatment group, $n=23(35.38 \%)$ failed to complete post-treatment questionnaires, regardless of how they adhered to treatment. In the waitlist group, $n=7(10.94 \%)$ failed to complete assessments after 10 weeks of waiting.

\section{Efficacy and Follow Up}

Means, standard deviations and effect sizes (Hedges' $g$ ) are displayed in Table 3. Symptom distress (BSI-18) decreased more in the treatment than in the waitlist group over time $(F=17.17, d f=2, p<.01)$. Treatment effects were also found for all secondary transdiagnostic and disorder-specific outcomes $(F=4.63-16.4, d f=1-2, p<.05)$. Betweengroup effect sizes ranged between $g=1.12$ (symptom distress) and $g=0.24$ (health anxiety) and within group effect sizes ranged between $g=1.38$ (symptom distress) and $g=0.32$ (social anxiety). Treatment gains for the transdiagnostic outcome measures - symptom distress (BSI18), positive and negative affect (PANAS), as well as satisfaction with life (SWLS) - were maintained at 1- and 6-month-follow-up $(F=0.16-1.22, d f=2, p>.05)$.

Table 3. Means and Standard Deviations/Errors for Primary and Secondary Outcome Measures and Within- and Between-Group Effect Sizes.

\begin{tabular}{|c|c|c|c|c|c|c|c|c|}
\hline \multirow{2}{*}{\multicolumn{2}{|c|}{ Measure }} & \multicolumn{2}{|c|}{ Treatment } & \multicolumn{2}{|c|}{ Waitlist } & \multicolumn{2}{|c|}{$\begin{array}{l}\text { Pre-Post Within ES } \\
\text { Hedges' } g \\
95 \% \mathrm{CI}\end{array}$} & \multirow[t]{2}{*}{$\begin{array}{c}\text { Between group ES } \\
\text { Hedges' } g \\
95 \% \text { CI }\end{array}$} \\
\hline & & $M$ & $S D / S E$ & $M$ & $S D / S E$ & Treatment & Waitlist & \\
\hline \multirow[t]{2}{*}{ BSI-18 } & Pre & 25.8 & 10.37 & 26.62 & 9.2 & -1.38 & -0.36 & -1.11 \\
\hline & Post & 13.11 & 1.37 & 23.09 & 1.25 & {$[-1.77,-1]$} & {$[-0.71,-0.01]$} & {$[-1.48,-0.74]$} \\
\hline
\end{tabular}




\begin{tabular}{|c|c|c|c|c|c|c|c|c|}
\hline & FU1 & 14.20 & 1.37 & & & & & \\
\hline & FU2 & 12.58 & 1.31 & & & & & \\
\hline \multirow{4}{*}{$\begin{array}{c}\text { PANAS } \\
\text { NA }\end{array}$} & Pre & 27.38 & 7.89 & 28.2 & 6.53 & -0.94 & -0.34 & -0.76 \\
\hline & Post & 20.54 & 1.04 & 25.81 & .95 & {$[-1.3,-0.58]$} & {$[-0.69,0]$} & {$[-1.11,-0.4]$} \\
\hline & FU1 & 20.62 & 1.06 & & & & & \\
\hline & FU2 & 20.20 & 1.02 & & & & & \\
\hline \multirow{4}{*}{$\begin{array}{c}\text { PANAS } \\
\text { PA }\end{array}$} & Pre & 20.11 & 5.15 & 19.03 & 5.0 & 0.79 & 0.3 & 0.70 \\
\hline & Post & 25.63 & .91 & 20.61 & .81 & {$[0.43,1.14]$} & {$[-0.05,0.65]$} & {$[0.35,1.06]$} \\
\hline & FU1 & 25.35 & 1.24 & & & & & \\
\hline & FU2 & 24.86 & 1.19 & & & & & \\
\hline \multirow[t]{4}{*}{ SWLS } & Pre & 15.64 & 5.9 & 15.45 & 6.16 & 0.46 & 0.07 & 0.39 \\
\hline & Post & 18.57 & .85 & 15.9 & .81 & {$[0.11,0.81]$} & {$[-0.28,0.42]$} & {$[0.05,0.74]$} \\
\hline & FU1 & 19.22 & 1.01 & & & & & \\
\hline & FU2 & 19.27 & 1.0 & & & & & \\
\hline \multirow[t]{2}{*}{ PHQ-9 } & Pre & 13.86 & 4.85 & 14.67 & 4.93 & -1.15 & -0.34 & -0.91 \\
\hline & Post & 8.2 & .74 & 12.9 & .66 & {$[-1.52,-0.78]$} & {$[-0.69,0.01]$} & {$[-1.27,-0.55]$} \\
\hline \multirow[t]{2}{*}{ GAD-7 } & Pre & 11.77 & 4.95 & 12.42 & 4.2 & -1.01 & -0.32 & -0.85 \\
\hline & Post & 7.18 & .67 & 10.97 & .59 & {$[-1.37,-0.64]$} & {$[-0.67,0.0]$} & {$[-1.21,-0.49]$} \\
\hline \multirow[t]{2}{*}{ LSAS } & Pre & 58.52 & 26.79 & 69.23 & 28.66 & -0.32 & -0.06 & -0.69 \\
\hline & Post & 50.33 & 3.59 & 67.67 & 3.4 & {$[-0.67,0.03]$} & {$[-0.4,0.29]$} & {$[-1.04,-0.33]$} \\
\hline \multirow[t]{2}{*}{ PAS } & Pre & 18.43 & 12.82 & 18.19 & 11.26 & -0.64 & -0.21 & -0.49 \\
\hline & Post & 10.72 & 1.64 & 15.9 & 1.49 & {$[-0.99,-0.29]$} & {$[-0.56,0.13]$} & {$[-0.84,-0.14]$} \\
\hline \multirow[t]{2}{*}{ SHAI } & Pre & 41.82 & 1.1 & 39.72 & 1.11 & -0.57 & -0.12 & -0.20 \\
\hline & Post & 36.41 & 1.36 & 38.45 & 1.3 & {$[-0.92,-0.22]$} & {$[-0.47,0.23]$} & {$[-0.55,0.14]$} \\
\hline \multirow[t]{2}{*}{ PHQ-15 } & Pre & 12.06 & 5.24 & 13.28 & 5.38 & -0.65 & -0.25 & -0.61 \\
\hline & Post & 8.62 & .76 & 11.91 & .69 & {$[-1.0,-0.3]$} & {$[-0.6,0.1]$} & {$[-0.97,-0.26]$} \\
\hline
\end{tabular}

Note. ES: Effect Size. BSI-18: Brief Symptom Inventory 18. PANAS NA: Negative Affect Subscale of the Positive and Negative Affect Schedule. PANAS PA: Positive Affect Subscale of the Positive and Negative Affect Schedule. SWLS: Satisfaction with Life Scale. PHQ-9: Patient Health Questionnaire 9. GAD-7: Generalized Anxiety Screener 7. LSAS: Liebowitz Social Anxiety Scale. PAS: Panic and Agoraphobia Scale. SHAI: Short Health Anxiety Inventory. PHQ-15: Patient Health Questionnaire

\section{Subgroup analyses}

Means, standard deviations and effect sizes (Hedges' $g$ ) for primary and secondary outcome measures in the anxiety, depression, and somatic symptom disorder subsample are displayed in Table 4. For the anxiety subsample, the interaction of time $\mathrm{x}$ group was significant for symptom distress, positive and negative affect, panic/agoraphobia, and depression $(F=3.54-6.37, d f=1-2, p<0.05)$. For the depressive subsample, the interaction of time x group was significant for symptom distress, positive and negative affect, depression, 
270 generalized anxiety, social anxiety, and health anxiety $(F=3.29-9.99, d f=1-2, p<.05)$. Due

271 to small sample size, the somatic symptom disorder sample was not analyzed separately. 
Table 4 Means in Primary Anxiety. Depressive and Somatic Symptom Disorders Subsamples and Effect Sizes in Anxious and Depressive subsample.

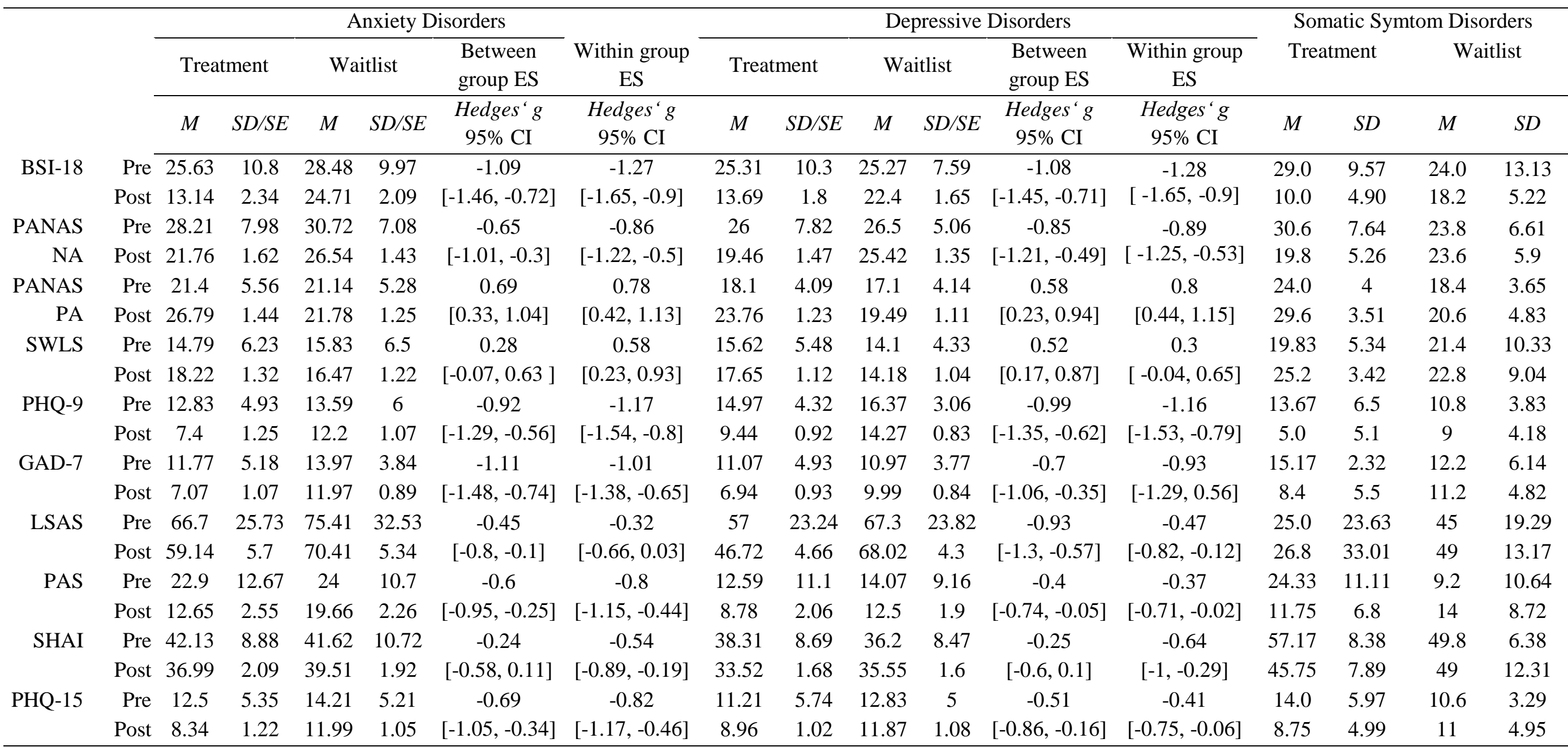

Note. ES: Effect Size. BSI-18: Brief Symptom Inventory 18. PANAS NA: Negative Affect Subscale of the Positive and Negative Affect Schedule. PANAS PA: Positive Affect Subscale of the Positive and Negative Affect Schedule. SWLS: Satisfaction with Life Scale. PHQ-9: Patient Health Questionnaire 9. GAD-7: Generalized Anxiety Screener 7. 
SAS: Liebowitz Social Anxiety Scale. PAS: Panic and Agoraphobia Scale. SHAI: Short Health Anxiety Inventory. PHQ-15: Patient Health Questionnaire 15. For the somatic symptom disorder subsample only observed means and standard deviations are reported because of small sample size 


\section{Response and Recovery Rates}

The majority of participants $(n=29 ; 69 \%)$ in the treatment group reliably improved, while $n=13$ (31\%) showed no reliable change. Nobody deteriorated. If participants who did not complete post-treatment assessments were classified as non-responders, the ratio of responders and non-responders would shift to $55 \%$ non-responders and $45 \%$ responders. Approximately half of the participants $(n=20 ; 48 \%)$ reliably recovered (falling below the Cutoff $C$ and depicting reliable change).

\section{Satisfaction with and Negative Effects of Treatment}

Overall, 84.62\% were "mostly" or "very" satisfied with treatment. Mean satisfaction was 3.27 out of $4(S D=0.62) .79 .49 \%$ of participants in the treatment group reported at least one negative treatment effect. Participants reported on average 2.54 negative treatment effects $(S D=2.78)$. "Unpleasant memories resurfaced" (38.46\%) and "I experienced more unpleasant feelings" (38.46\%) showed the highest frequency. Items associated with elevated symptoms ("symptom factor") showed the highest mean $(M=2.31, S D=3.18)$. Participants felt that the negative effects affected their well-being slightly to moderately $(M=1.39, S D=0.58)$.

\section{Diagnostic Status}

We reached $n=37(57.81 \%)$ of the treatment group for the post-treatment structured clinical interview. The mean number of total diagnoses dropped to $0.81(S D=1.08)$. The majority of participants did not fulfill criteria for their primary diagnosis $(n=26 ; 70.27 \%)$. In regard to comorbidity, $n=24(64.86 \%)$ did not fulfill criteria for comorbid diagnoses. The number of comorbid diagnoses at post-intervention ranged from 0 to 3 . If participants who we did not reach for the post-treatment interview experienced no effect on their primary diagnosis, $59.3 \%$ of participants would be unchanged, while $40.6 \%$ of participants did not fulfill criteria of their primary diagnosis. 
This study investigated the effects of a guided Internet-based transdiagnostic intervention based on the UP. Overall, the intervention yielded large reductions on symptom distress and medium to large effects on negative and positive affect, as well as anxiety and depression. Small effects were observed for satisfaction with life and health anxiety. The majority of participants exhibited reliable change. These results are comparable both to research on the UP's efficacy as a face-to-face and Internet-based intervention, as well as other (transdiagnostic) Internet-based treatments $[10,15-17,59]$. The transdiagnostic intervention's impact on comorbidity is of special interest. The mean number of diagnoses in the fairly comorbid sample (78\% comorbidity) dropped from almost 3 (range 1-8) to under 1 (range 0-3) which is very comparable to other face-to-face trials on the UP [15,60].

Our findings substantiate Tulbure and colleagues' (2018) findings on the efficacy of the UP when applied online. Both interventions were highly similar in length and content and produced comparable results. The degree of comorbidity, prior psychotherapy, and current medication was higher in our sample compared to the other Internet-based UP [17]. This suggests that an Internet-delivered UP can be expanded to a comorbid and impaired population. Subgroup analyses also revealed that the intervention in our study produced similar effects in participants with primary anxiety or depression. While preliminary, these results are encouraging of the UP's potential to treat depression.

Beyond symptom improvements, other indicators are important determining factors for the uptake of interventions. About $75 \%$ of participants completed the core modules of treatment. While this adherence rate is not unusual in Internet-based settings [61], higher adherence may have benefitted outcomes, especially considering that less than half of participants completed all modules [10]. Usage data suggested that participants who chose to work with the program engaged with it actively. The majority of participants was mostly satisfied to very satisfied with the program but also reported experiencing negative effects. That approximately $80 \%$ of participants report negative effects is higher than reports from 
other trials (56-65\%) [48]. Negative effects are not synonymous to unwanted effects necessarily - that participants experience more negative emotions short-term can even be an intended effect in a program focused on reducing avoidance and experiencing emotions.

Our results need to be interpreted in the light of several limitations: The trial suffered from an attrition rate of $35 \%$ of participants who did not provide post-treatment data. High attrition rates like this are not uncommon in Internet-based treatments [62] but they can limit the validity and reliability of findings and could be indicative of limited acceptability of the intervention. Subgroup analyses of the anxiety and depression subsamples revealed that not all symptom measures showed significant effects, likely due to small sample sizes and limited statistical power related to dropout. We failed to recruit a sufficient sample of participants with somatic symptom disorders within the study's timeframe. Recruitment via primary health care providers might be a more fruitful recruitment strategy for this population [e.g., 63]. The self-selection of participants and the predominantly female, highly educated, and psychotherapy-experienced sample does not allow for a generalization of results.

Overall, these results strengthen the evidence base of the UP as a viable treatment option beyond anxiety disorders and demonstrate that the UP can successfully be delivered over the Internet over the course of ten weeks. Applying a transdiagnostic intervention online can help to overcome barriers to treatments and accelerate the dissemination of evidencebased treatments for a broad variety of the most common disorders. However, dropout and attrition rates suggest that several modifications should be made to the intervention before employing it, e.g. by further simplifying it, delivering it modularly [64,65], or implementing monitoring to detect participants at risk of treatment failure [66]. While a waitlist control group is a necessary first step to establish efficacy, the intervention should next be measured against an active control condition, e.g. against disorders-specific treatments. The UP aims at changing transdiagnostic processes - shedding light on whether changes in those processes 
350 are relevant for symptom change is an important next research endeavor to further our

351 understanding beyond the treatment's efficacy.

352 
353

354

355

356

357

358

359

360

361

362

363

364

365

366

367

368

369

370

371

372

373

374

375

\section{Data Availability Statement}

De-identified data that support the findings of this study are available on OSF at 10.17605/OSF.IO/7AW3V.

\section{Author Contribution}

Conceptualization: Carmen Schaeuffele, Christine Knaevelsrud, Babette Renneberg, Johanna Boettcher

Data Curation: Sophie Homeyer, Luis Perea, Lisa Scharf

Formal Analysis: Carmen Schaeuffele

Investigation: Sophie Homeyer, Luis Perea, Lisa Scharf

Methodology: Carmen Schaeuffele, Ava Schulz, Johanna Boettcher

Project Administration: Carmen Schaeuffele

Supervision: Ava Schulz, Christine Knaevelsrud, Babette Renneberg

Visualization: Carmen Schaeuffele

Writing - Original Draft Preparation: Carmen Schaeuffele

Writing - Review \& Editing: Carmen Schaeuffele, Sophie Homeyer, Luis Perea, Lisa

Scharf, Ava Schulz., Christine Knaevelsrud, Babette Renneberg, Johanna Boettcher 
1. Jacobi F, Höfler M, Siegert J, Mack S, Gerschler A, Scholl L, et al. Twelve-month prevalence, comorbidity and correlates of mental disorders in Germany: The Mental Health Module of the German Health Interview and Examination Survey for Adults (DEGS1-MH). Int J Methods Psychiatr Res. 2014;23: 304-19. doi:10.1002/mpr.1439

2. Kessler RC, Chiu WT, Demler O, Walters EE. Prevalence, severity, and comorbidity of 12-month DSM-IV disorders in the national comorbidity survey replication. Arch Gen Psychiatry. 2005;62: 617-627. doi:10.1001/archpsyc.62.6.617

3. Klein Hofmeijer-Sevink M, Batelaan NM, van Megen HJGM, Penninx BW, Cath DC, van den Hout MA, et al. Clinical relevance of comorbidity in anxiety disorders: A report from the Netherlands Study of Depression and Anxiety (NESDA). J Affect Disord. 2012;137: 106-112. doi:10.1016/j.jad.2011.12.008

4. Rapaport MH, Clary C, Fayyad R, Endicott J. Quality-of-life impairment in depressive and anxiety disorders. Am J Psychiatry. 2005;162: 1171-1178. doi:10.1176/appi.ajp.162.6.1171

5. Cuijpers P. Targets and outcomes of psychotherapies for mental disorders: an overview. World Psychiatry. 2019;18: 276-285. doi:10.1002/wps.20661

6. Sauer-Zavala S, Gutner CA, Farchione TJ, Boettcher HT, Bullis JR, Barlow DH. Current definitions of "transdiagnostic" in treatment development: A search for consensus. Behav Ther. 2017;48: 128-138. doi:https://doi.org/10.1016/j.beth.2016.09.004

7. Newby JM, McKinnon A, Kuyken W, Gilbody S, Dalgleish T. Systematic review and meta-analysis of transdiagnostic psychological treatments for anxiety and depressive disorders in adulthood. Clin Psychol Rev. 2015;40: 91-110. doi:10.1016/j.cpr.2015.06.002

8. Pearl SB, Norton PJ. Transdiagnostic versus diagnosis specific cognitive behavioural therapies for anxiety: A meta-analysis. J Anxiety Disord. 2017;46: 11-24. doi:10.1016/j.janxdis.2016.07.004

9. Newby JM, Twomey C, Yuan Li SS, Andrews G. Transdiagnostic computerised cognitive behavioural therapy for depression and anxiety: A systematic review and meta-analysis. J Affect Disord. 2016;199: 30-41. doi:10.1016/j.jad.2016.03.018

10. Păsărelu CR, Andersson G, Bergman Nordgren L, Dobrean A. Internet-delivered transdiagnostic and tailored cognitive behavioral therapy for anxiety and depression: a systematic review and meta-analysis of randomized controlled trials. Cogn Behav Ther. 2017;46: 1-28. doi:10.1080/16506073.2016.1231219

11. Schaeuffele C, Schulz A, Knaevelsrud C, Renneberg B, Boettcher J. CBT at the crossroads: The rise of transdiagnostic treatments. Int J Cogn Ther. 2020. doi:10.1007/s41811-020-00095-2

12. Cassiello-Robbins C, Southward MW, Tirpak JW, Sauer-Zavala S. A systematic review of Unified Protocol applications with adult populations: Facilitating widespread dissemination via adaptability. Clin Psychol Rev. 2020;78: 101852. doi:10.1016/j.cpr.2020.101852

13. Barlow DH, Allen LB, Choate ML. Toward a unified treatment for emotional disorders. Behav Ther. 2004;35: 205-230. doi:10.1016/S0005-7894(04)80036-4 
14. Ellard KK, Fairholme CP, Boisseau CL, Farchione TJ, Barlow DH. Unified protocol for the transdiagnostic treatment of emotional disorders: Protocol development and initial outcome data. Cogn Behav Pract. 2010; 88-101. doi:10.1016/j.cbpra.2009.06.002

15. Barlow DH, Farchione TJ, Bullis JR, Gallagher MW, Murray-Latin H, Sauer-Zavala S, et al. The Unified Protocol for transdiagnostic treatment of emotional disorders compared with diagnosis-specific protocols for anxiety disorders: A randomized clinical trial. JAMA Psychiatry. 2017. doi:10.1001/jamapsychiatry.2017.2164

16. Farchione TJ, Fairholme CP, Ellard KK, Boisseau CL, Thompson-Hollands J, Carl JR, et al. Unified protocol for transdiagnostic treatment of emotional disorders: A randomized controlled trial. Behav Ther. 2012;43: 666-678. doi:10.1016/j.beth.2012.01.001

17. Tulbure BT, Rusu A, Sava FA, Sălăgean N, Farchione TJ. A web-based transdiagnostic intervention for affective and mood disorders: Randomized controlled trial. JMIR Ment Health. 2018;5: 36. doi:10.2196/mental.8901

18. Sakiris N, Berle D. A systematic review and meta-analysis of the Unified Protocol as a transdiagnostic emotion regulation based intervention. Clin Psychol Rev. 2019;72: 101751. doi:10.1016/j.cpr.2019.101751

19. Boswell JF, Anderson LM, Barlow DH. An idiographic analysis of change processes in the unified transdiagnostic treatment of depression. J Consult Clin Psychol. 2014;82: 1060-71. doi:10.1037/a0037403

20. Boswell JF, Bugatti M. An exploratory analysis of the impact of specific interventions: Some clients reveal more than others. J Couns Psychol. 2016;63: 710-720. doi:10.1037/cou0000174

21. Farchione TJ, Boswell JF, Wilner JG. Behavioral activation strategies for major depression in transdiagnostic cognitive-behavioral therapy: An evidence-based case study. Psychotherapy. 2017;54: 225-230. doi:10.1037/pst0000121

22. Hague B, Scott S, Kellett S. Transdiagnostic CBT treatment of co-morbid anxiety and depression in an older adult: Single case experimental design. Behav Cogn Psychother. 2015;43: 119-124. doi:10.1017/S1352465814000411

23. Ito $\mathrm{M}$, Horikoshi $\mathrm{M}$, Kato $\mathrm{N}, \mathrm{Oe} \mathrm{Y}$, Fujisato $\mathrm{H}$, Nakajima $\mathrm{S}$, et al. Transdiagnostic and transcultural: Pilot study of unified protocol for depressive and anxiety disorders in Japan. Behav Ther. 2016;47: 416-430. doi:10.1016/j.beth.2016.02.005

24. Tyrer $\mathrm{P}$, Eilenberg $\mathrm{T}$, Fink $\mathrm{P}$, Hedman $\mathrm{E}$, Tyrer $\mathrm{H}$. Health anxiety: The silent, disabling epidemic. BMJ (Clinical research ed.). 2016. p. 2250. doi:10.1136/bmj.i2250

25. Margraf J, Cwik JC, Pflug V, Schneider S. Strukturierte klinische Interviews zur Erfassung psychischer Störungen über die Lebensspanne. Z Für Klin Psychol Psychother. 2017;46: 176186. doi:10.1026/1616-3443/a000430

26. Margraf J, Cwik JC, Suppiger A, Schneider S. DIPS open access: Diagnostic interview for mental disorders. [DIPS Open Access: Diagnostisches Interview bei psychischen Störungen.]. Bochum: Mental Health Research and Treament Center, Ruhr-Universität Bochum; 2017.

27. Derogatis LR. BSI-18: Brief Symptom Inventory 18 - Administration, scoring, and procedures manual. Minneapolis: NCS Pearson; 2000. 
28. Franke GH, Ankerhold A, Haase M, Jäger S, Tögel C, Ulrich C, et al. Der Einsatz des Brief Symptom Inventory 18 (BSI-18) bei Psychotherapiepatienten. Psychother Psychosom Med Psychol. 2011;61: 82-6. doi:10.1055/s-0030-1270518

29. Derogatis LR, Fitzpatrick M. The SCL-90-R, the Brief Symptom Inventory (BSI), and the BSI-18. The use of psychological testing for treatment planning and outcomes assessment: Instruments for adults. Lawrence Erlbaum Associates Publishers; 2004.

30. Prinz U, Nutzinger DO, Schulz H, Petermann F, Braukhaus C, Andreas S. Comparative psychometric analyses of the SCL-90-R and its short versions in patients with affective disorders. BMC Psychiatry. 2013;13: 104. doi:10.1186/1471-244X-13-104

31. Krohne HW, Egloff B, Kohlmann C-W, Tausch A. Untersuchungen mit einer deutschen Version der "Positive and Negative Affect Schedule" (PANAS). Diagnostica. 1996. pp. 139-156.

32. Watson D, Clark LA, Tellegen A. Development and validation of brief measures of positive and negative affect: The PANAS scales. Journal of Personality and Social Psychology. 1988. pp. 1063-1070. doi:10.1037//0022-3514.54.6.1063

33. Diener E, Emmons RA, Larsen RJ, Griffin S. The satisfaction with life scale. J Pers Assess. 1985;49: 71-75. doi:https://doi.org/10.1207/s15327752jpa4901_13

34. Glaesmer H, Grande G, Braehler E, Roth M. The German Version of the Satisfaction With Life Scale (SWLS). European Journal of Psychological Assessment. 2011. pp. 127-132. doi:10.1027/1015-5759/a000058

35. Kroenke K, Spitzer RL. The PHQ-9: a new depression diagnostic and severity measure. Psychiatric annals. 2002. pp. 509-515.

36. Lowe B. Comparative validity of three screening questionnaires for DSM-IV depressive disorders and physicians? diagnoses. J Affect Disord. 2004;78: 131-140. doi:10.1016/S01650327(02)00237-9

37. Löwe B, Decker O, Müller S, Brähler E, Schellberg D, Herzog W, et al. Validation and standardization of the Generalized Anxiety Disorder Screener (GAD-7) in the general population. Medical care. 2008. pp. 266-74. doi:10.1097/MLR.0b013e318160d093

38. Spitzer RL, Kroenke K, Williams JBW, Löwe B. A brief measure for assessing generalized anxiety disorder: the GAD-7. Archives of internal medicine. 2006. pp. 1092-7. doi:10.1001/archinte.166.10.1092

39. Heimberg RG, Horner KJ, Juster HR, Safren SA, Brown EJ, Schreiner FR, et al. Psychometric properties of the Liebowitz Social Anxiety Scale. Psychological medicine. 1999. pp. 199-212.

40. Stangier U, Heidenreich T. Die Liebowitz Soziala Angst-Skala (LSAS). Collegium Internationale Psychiatriae Scalarum, editor. Internationale Skalen für Psychiatrie. Göttingen: Beltz; 2005.

41. Fresco DM, Coles ME, Heimberg RG, Liebowitz MR, Hami S, Stein MB, et al. The Liebowitz Social Anxiety Scale: a comparison of the psychometric properties of self-report and clinicianadministered formats. Psychol Med. 2001;31: 1025-1035. doi:10.1017/S0033291701004056

42. Bandelow B. Assessing the efficacy of treatments for panic disorder and agoraphobia. II. The Panic and Agoraphobia Scale. Int Clin Psychopharmacol. 1995;10: 73-82. 
43. Bandelow B. PAS - Panik- und Agoraphobie-Skala: Manual. Göttingen: Hogrefe; 2016.

44. Bailer J, Rist F, Müller T, Mier D, Diener C, Ofer J, et al. Erfassung von Krankheitsangst mit dem Short Health Anxiety Inventory (SHAI). Verhal Verhal. 2013;34: 378-398.

45. Salkovskis PM, Rimes KA, Warwick HMC, Clarke DM. The Health Anxiety Inventory: Development and validation of scales for the measurement of health anxiety and hypochondriasis. Psychological Medicine. 2002. doi:10.1017/S0033291702005822

46. Gräfe K, Zipfel S, Herzog W, Löwe B. Screening psychischer Störungen mit dem "Gesundheitsfragebogen für Patienten (PHQ-D)". Diagnostica. 2004;50: 171-181. doi:10.1026/0012-1924.50.4.171

47. Kroenke K, Spitzer RL, Williams JBW. The PHQ-15: Validity of a new measure for evaluating the severity of somatic symptoms. Psychosom Med. 2002;64: 258-66. doi:https://doi.org/10.1097/00006842-200203000-00008

48. Rozental A, Kottorp A, Forsström D, Månsson K, Boettcher J, Andersson G, et al. The Negative Effects Questionnaire: psychometric properties of an instrument for assessing negative effects in psychological treatments. Behav Cogn Psychother. 2019;47: 559-572. doi:10.1017/\$1352465819000018

49. Attkisson CC, Zwick R. The client satisfaction questionnaire. Eval Program Plann. 1982;5: 233237. doi:10.1016/0149-7189(82)90074-X

50. Schmidt J, Lamprecht F, Wittmann WW. Zufriedenheit mit der stationären Versorgung. Entwicklung eines Fragebogens und erste Validitätsuntersuchungen. Psychother Psychosom Med Psychol. 1989;39: 248-255.

51. Kriz D, Nübling R, Steffanowski A, Wittmann WW, Schmidt J. Patientenzufriedenheit in der stationären Rehabilitation: Psychometrische Reanalyse des ZUF-8 auf der Basis multizentrischer Stichproben verschiedener Indikation. Z Für Med Psychol. 2008; 67-79.

52. Faul F, Erdfelder E, Lang A-G, Buchner A. G*Power 3: A flexible statistical power analysis program for the social, behavioral, and biomedical sciences. Behav Res Methods. 2007;39: 175191. doi:10.3758/BF03193146

53. R Core Team. R: A language and environment for statistical computing. Vienna, Austria: $\mathrm{R}$ Foundation for Statistical Computing; 2013. Available: http://www.R-project.org/

54. Bates D, Mächler M, Bolker B, Walker S. Fitting linear mixed-effects models using Ime4. ArXiv14065823 Stat. 2014 [cited 8 Apr 2020]. Available: http://arxiv.org/abs/1406.5823

55. Kuznetsova A, Brockhoff PB, Christensen RHB. ImerTest package: Tests in linear mixed effects models. J Stat Softw. 2017;82. doi:10.18637/jss.v082.i13

56. Jacobson NS, Truax P. Clinical Significance: A statistical approach to defining meaningful change in psychotherapy research. J Consult Clin Psychol. 1991;59: 12-19.

doi:https://doi.org/10.1037/10109-042

57. Lambert MJ, Ogles BM. Using clinical significance in psychotherapy outcome research: The need for a common procedure and validity data. Psychother Res. 2009;19: 493-501.

doi:10.1080/10503300902849483 
58. Franke GH, Jaeger S, Glaesmer H, Barkmann C, Petrowski K, Braehler E. Psychometric analysis of the brief symptom inventory 18 (BSI-18) in a representative German sample. BMC Med Res Methodol. 2017;17: 14. doi:https://doi.org/10.1186/s12874-016-0283-3

59. Rozental A, Andersson G, Carlbring P. In the absence of effects: An individual patient data metaanalysis of non-response and its predictors in Internet-based cognitive behavior therapy. Front Psychol. 2019;10. doi:10.3389/fpsyg.2019.00589

60. Steele SJ, Farchione TJ, Cassiello-Robbins C, Ametaj A, Sbi S, Sauer-Zavala S, et al. Efficacy of the Unified Protocol for transdiagnostic treatment of comorbid psychopathology accompanying emotional disorders compared to treatments targeting single disorders. J Psychiatr Res. 2018;104: 211-216. doi:10.1016/j.jpsychires.2018.08.005

61. van Ballegooijen W, Cuijpers P, van Straten A, Karyotaki E, Andersson G, Smit JH, et al. Adherence to Internet-based and face-to-face cognitive behavioural therapy for depression: A meta-analysis. PLoS ONE. 2014;9. doi:10.1371/journal.pone.0100674

62. Christensen $\mathrm{H}$, Griffiths KM, Farrer L. Adherence in Internet interventions for anxiety and depression: Systematic review. J Med Internet Res. 2009;11: e13. doi:10.2196/jmir.1194

63. Hedman E, Axelsson E, Andersson E, Lekander M, Ljótsson B. Exposure-based cognitivebehavioural therapy via the Internet and as bibliotherapy for somatic symptom disorder and illness anxiety disorder: Randomised controlled trial. Br J Psychiatry J Ment Sci. 2016;209: 407413. doi:10.1192/bjp.bp.116.181396

64. Fisher AJ, Bosley HG, Fernandez KC, Reeves JW, Soyster PD, Diamond AE, et al. Open trial of a personalized modular treatment for mood and anxiety. Behaviour research and therapy. Behav Res Ther. 2019;116: 69-79. doi:https://doi.org/10.1016/j.brat.2019.01.010

65. Sauer-Zavala S, Cassiello-Robbins C, Ametaj AA, Wilner JG, Pagan D. Transdiagnostic treatment personalization: The feasibility of ordering unified protocol modules according to patient strengths and weaknesses. Behav Modif. 2018; 518-543. doi:10.1177/0145445518774914

66. Forsell E, Jernelöv S, Blom K, Kraepelien M, Svanborg C, Andersson G, et al. Proof of concept for an adaptive treatment strategy to prevent failures in Internet-delivered CBT: A single-blind randomized clinical trial with insomnia patients. Am J Psychiatry. 2019;176: 315-323. doi:10.1176/appi.ajp.2018.18060699 